\title{
LETTER
}

\section{Diagnostic criteria and treatment protocol for post-burn sepsis}

\author{
Peng Yizhi, Chen Jing, Yuan Zhiqiang, Li Xiaolu, Luo Gaoxing and Wu Jun* on behalf of Editorial Board of \\ Guidelines for the Treatment of Burn Infection, Chinese Medical Association
}

The diagnostic criteria of sepsis have been in accordance with those of the systemic inflammatory response syndrome for decades [1]. Scholars find them inadequate, however, especially for burn patients. After discussion by burn experts in China [2], the following diagnostic criteria and therapy guidelines for sepsis are suggested.

\section{Diagnostic criteria}

Preliminary diagnosis of post-burn sepsis can be made if six out of the first 11 criteria below are met. This preliminary diagnosis can be confirmed if any one aspect described in the last criterion is met.

The diagnostic criteria are: (1) mental excitement, hallucinations, disorientation or depression; (2) abdominal distension, diminished bowel sound; (3) rapidly deteriorated burn wounds, exhibited as wet, dark and/or deepened wounds with necrotic spots, and so forth; (4) core temperature $>39.0^{\circ} \mathrm{C}$ or $<36.5^{\circ} \mathrm{C}$; (5) increased heart rate - adults $>130$ times/minute, children of all ages $>2$ standard deviations of normal value; (6) increased respiratory rate - adults $>28$ times/minute (without mechanical ventilation), children of all ages $>2$ standard deviations of normal value; (7) thrombocytopenia adults $<50 \times 10^{9} / \mathrm{l}$, children of all ages $<2$ standard deviations of normal value; (8) peripheral white blood cell count - adults $>15 \times 10^{9} / 1$ or $<5 \times 10^{9} / \mathrm{l}$, in which neutrophil percentage $>80 \%$ or immature granulocytes $>10 \%$, children of all ages $>2$ or $<2$ standard deviations of normal value; (9) blood procalcitonin $>0.5 \mu \mathrm{g} / \mathrm{l}$;
(10) blood sodium levels $>155 \mathrm{mmol} / \mathrm{l}$; (11) blood glucose $>14 \mathrm{mmol} / \mathrm{l}$ (no history of diabetes); and (12) positive blood culture or positive response to antibiotic therapy.

\section{Treatment guidelines}

Recommended guidelines include infectious source control, rational use of antibiotics, continuous blood purification, application of glucocorticoids, immunomodulation, symptomatic and supportive treatment, and prevention of hospital-acquired infection.

\section{Competing interests}

The authors declare that they have no competing interests.

\section{Acknowledgements}

This work was supported by the Specific Project of Health, Ministry of Health of China (No. 201202002), by a grant from the CPLA Scientific Research Fund (No. BWS11 J039), and by the Key Project on Advanced Clinical Technology for Military Hospital (No. 2010gxjs068).

Published: 18 January 2013

References

1. Bone RC, Balk RA, Cerra FB, Dellinger RP, Fein AM, Knaus WA, Schein RM, Sibbald WJ: Definitions for sepsis and organ failure and guidelines for the use of innovative therapies in sepsis. The ACCP/SCCM Consensus Conference Committee. American College of Chest Physicians/Society of Critical Care Medicine. Chest 1992, 101:1644-1655.

2. Peng $Y Z$, Yuan ZQ: [Standardized definitions and diagnosis criteria for infection in burn patients]. Chin J Burns 2007, 23:404-405.

doi:10.1186/cc11912

Cite this article as: Yizhi P, et al.: Diagnostic criteria and treatment protocol for post-burn sepsis. Critical Care 2013, 17:406.
*Correspondence: junwupro1@yahoo.com.cn

State Key Laboratory of Trauma, Burns and Combined Injury, Chongqing Key Laboratory for Proteomics Disease, Institute of Burn Research, Southwest Hospital, the Third Military Medical University, Chongqing 400038, China 